\title{
Appropriate methods to analyse Power Conversion Harmonics
}

\author{
P. Verzele, J. Knockaert and J. Desmet \\ Technical University College Howest, Ghent University \\ Graaf Karel de Goedelaan 34, 8500 Kortrijk (Belgium) \\ Phone number: +0032 56/24 12 35, Fax number: +0032 56/ 241234 \\ e-mail: pauline.verzele@howest.be, jos.knockaert@howest.be, jan.desmet@howest.be
}

\begin{abstract}
Nowadays, non-linear loads represent the majority of the residential electrical consumers. The limits on emission and immunity are imposed by IEC- standards, however there is a lack in the domain $2-150 \mathrm{kHz}$. Where power quality standards focus on the current, EMC standards use voltage limits. An appropriate method for measuring high frequency grid disturbances is explored. Measurement techniques described by the existing standards for power quality and EMC are investigated. The aim of this work is to find a robust measurement method for the considered frequency range $2-150 \mathrm{kHz}$. Experimental results are presented in order to validate the analyzed methods.
\end{abstract}

\section{Key words}

Power Quality, Interference, Measurement Techniques

\section{Introduction}

Last decade, a massive shift in consumer topology has been noticed in low voltage distribution networks. Modern households contain computers, flat screen TV's, induction cookers etc. One of the most obvious changes is the shift from incandescent lamps to compact fluorescent lamps (CFL's) and luminaires with light emitting diodes (LED's). These modern types of electric devices contain switched mode power supplies (SMPS) which are a source of high frequency disturbances. Additionally, the resistive behavior of the low voltage distribution network changes into a more capacitive behavior. As a result a malfunction of equipment and disturbed power line communication can occur [1][2]. Besides the well- known low frequent harmonic current distortion and EMI problems, also medium frequency conducted disturbances will occur. The latter emerge in the frequency range $2-150 \mathrm{kHz}$ and are further called 'Power Conversion Harmonics' (PCH). In this paper, both methods to measure and quantify $\mathrm{PCH}$ are investigated. Therefore, a study of the measurement methods mentioned in the standards for Power Quality and EMC is made. Next, some alternative measurement techniques are studied in detail. Measurements are performed in order to test the robustness of the different methods with respect to the $\mathrm{PCH}$ and are validated by comparative measurements.

\section{Power Quality measurements}

In the frequency range up to $2 \mathrm{kHz}\left(40^{\text {th }}\right.$ harmonic in a $50 \mathrm{~Hz}$ power system), IEC 61000-3-2 [3] defines limits for harmonic current emission. Limits are set as current values given at a certain harmonic order. Additional requirements for the test conditions (e.g. supply voltage) guarantee standardized and reproducible measurements. Testing and measurement techniques are given in IEC 61000-4-7 [4]. Instrumentation intended for measuring spectral components in the frequency range up to $9 \mathrm{kHz}$ is discussed and in an informative annex, measurements above $9 \mathrm{kHz}$ are tentatively defined. The instrumentation used to measure harmonic emission mostly use the discrete Fourier transform (DFT) to capture the harmonic content of a signal, although other principles are not excluded by the standard. Two classes of accuracy are allowed in instrumentation for measuring harmonic components, although a distinction in the application is made. Class 1 instruments are the most precise instruments and are recommended for emission measurements. The accuracy of Class 2 equipment is lower and as a consequence, this type of devices is less expensive. In case a Class 2 device is used for emission measurements, the measured values should be lower than $90 \%$ of the allowed limits due to the increased uncertainty in comparison with a Class 1 device.

\section{EMC measurements}

CISPR standards define limits starting from $150 \mathrm{kHz}$, e.g. CISPR 22 [5] defines limits and methods of measurement on information technology equipment, while CISPR 14-1 [6] gives requirements for household appliances, electric tools and similar equipment. One exception is CISPR15 [7], which defines provisional limits in the $\mathrm{PCH}$ domain. The CISPR standards impose the use of an artificial mains network (AMN) (also called line impedance stabilization network (LISN)) and a measuring receiver to quantify conducted emissions. An AMN has three important functions. It provides a defined output impedance seen from the equipment under test (EUT), while unwanted radio frequency signals on the supply mains are blocked. Additionally, the disturbance voltage 
coming from the EUT is coupled into the EMI receiver. When the AMN cannot be used without unduly influencing the EUT or the test equipment, a voltage probe can be used. As the voltage probe is a passive probe, the major drawback is the large attenuation factor of more than $30 \mathrm{~dB}$. For more specifications about this $A M N$, voltage probe, measuring receiver and other devices used in EMC measurements, the CISPR standards refer to CISPR 16 [8]. In this standard, five variations of the AMN are described (e.g. $50 \Omega / 50 \mu \mathrm{H}+5 \Omega$ ). The appropriate one depends on the considered frequency range. The EMI receiver is connected to the AMN in order to measure levels of conducted disturbances. As the EMI receiver has a fixed input impedance of $50 \Omega$, high frequency currents are measured as a voltage over the $50 \Omega$ - resistor.

\section{Comparative study}

Power quality standards impose the measurement of the current, given several constraints for the test voltage. As a consequence, this voltage should also be measured and controlled. However, in the standard IEC 61000-3-2 [3], nothing is mentioned about the source impedance and the angles of the voltage harmonics [9]. From Figure 1 it can be seen that next to the harmonic content of the test voltage, the source impedance is of great importance, especially for higher order harmonics.

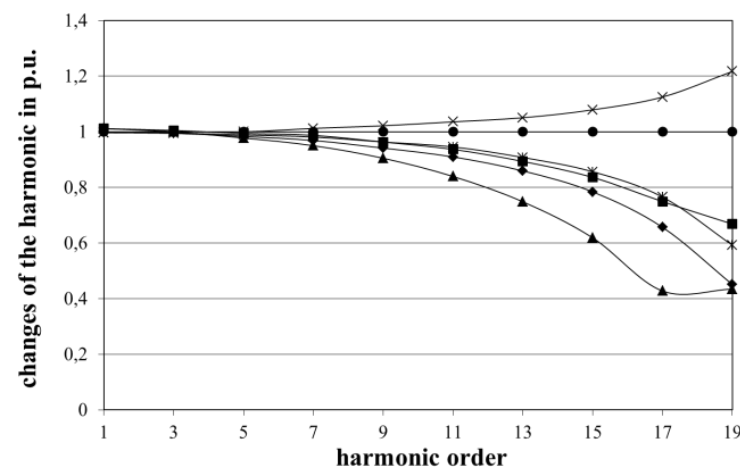

Figure 1: Influence of harmonic spectrum of testing voltage and source impedance during compliance test with respect to standard requirements for supply voltages and impedances [9]

When considering EMC measurement techniques, an AMN is used. Where power quality measurements apply restrictions on the test voltage, EMI measurements apply restrictions on the impedance. This means that the output impedance seen by the EUT is defined, which is not the case for power quality measurements. Seen the high influence of the measuring impedance for higher order harmonics (Figure 1), the use of an AMN is preferred for the analysis of $\mathrm{PCH}$. Whether $\mathrm{PCH}$ have to be measured as a voltage (over the $50 \Omega$ resistor of the measurement receiver) or as a current (using current probes) is studied in the next section.

\section{PCH measurements}

\section{A. General considerations}

Taking into consideration the previous paragraphs, it is obvious that the range $2 \mathrm{kHz}$ to $9 \mathrm{kHz}$ and by extension 9 to $150 \mathrm{kHz}$ is up till now a rather unconsidered area. Where the range beneath $2 \mathrm{kHz}$ normally uses current measurements, the range above $150 \mathrm{kHz}$ uses voltage measurements. This gives rise to the question what the available measurements methods are in the range $2-150$ $\mathrm{kHz}$. The choice for a correct measuring probe depends on different parameters.

The primary parameter to consider is the bandwidth. The frequency under consideration is between $2 \mathrm{kHz}$ and 150 $\mathrm{kHz}$. Typical current probes based on current transformers have a sharp roll-off in the frequency band beneath some Hz. This is no problem in this case. At the upper limit of this range, it can be interesting to place an anti-aliasing filter, preventing frequencies at higher ranges to mirror to the frequency interval under consideration. The second parameter is the sensitivity. Where harmonics require a measurement from as low as $\mathrm{mA}$ up to hundreds of $\mathrm{A}, \mathrm{PCH}$ are in the range $10 \mu \mathrm{A}$ to tens of $\mathrm{A}$. This requires a largely increased sensitivity while keeping an equal dynamic range. Third problem while performing measurements is the environmental influence. For reproducible measurements, the influence of interfering parameters has to be cancelled. Within the considered frequency range, the following issues can have an influence:

- High frequency interference (above $150 \mathrm{kHz}$ ) from the EUT

- High frequency interference from the power supply

- Low frequency interference (DC to $150 \mathrm{kHz}$ ) due to inductive coupling

- Low frequency interference from the power supply

Influence of the grid impedance

When a non-filtered power supply is used, this supply can contain high frequency components. This can cause problems directly into the considered frequency range or indirectly by aliasing. Several solutions are possible. When the interference is caused by the grid, a filtered supply can be used. If the interference is caused by the EUT in combination with aliasing, an anti-aliasing filter can be used. In practice, a null measurement is performed before and after the measurement. Low frequency interference can be caused by inductive coupling by neighboring power lines. A special type of low frequency interference is the fundamental of the current of the EUT which saturates the core of the current probe.

\section{B. Artificial mains networks between 2 and $150 \mathrm{kHz}$}

In CISPR16-1 [8], several types of AMN are defined. The topology is similar, but the values of the used components are different. This makes the AMN suitable for frequencies between $9 \mathrm{kHz}$ and $100 \mathrm{MHz}$. For the lower frequency range, the standard EN 50065 [10] mentions a modified AMN suitable between $3 \mathrm{kHz}$ and 9 $\mathrm{kHz}$ (Figure 2). This standard is similar to IEC 61000-3-8 [11]. 




Figure 2: AMN $3 \mathrm{kHz}-9 \mathrm{kHz}[11]$

\section{Current measurements}

Current probes can be divided in types using Hall-sensors and current transformers. The main difference between these two types is the ability to go down to DC. DCcurrent measurements are not possible for current transformer based probes. Current probes are normally used as diagnostic tool to measure the common mode current. They benefit from the ability to be clamped on a cable or wire. Figure 3 shows a probe ranging from $20 \mathrm{~Hz}$ - $100 \mathrm{MHz}$. The current in the considered range 2 to 150 $\mathrm{kHz}$ are typically differential mode currents. This can be measured with this type of probe if the current does not drive the probe into saturation. The sensitivity can be a problem for this type of probe in the considered range. Current probes are specially made to have a flat response in the high frequency range, but have a sharp roll-off in the low frequency range. The main parameter to consider is the transfer impedance.

$$
i=\frac{v}{z_{t}}
$$

This impedance $z_{t}$ gives the relation between the measured voltage $v$ by the receiver and current $i$ in the wire. The datasheets normally mentions the transfer impedance as a logarithmic value:

$$
i_{d b \mu A}=v_{d B \mu V}-z_{t_{-} d B \Omega}
$$

As this transfer impedance increases very fast below 150 $\mathrm{kHz}$, the sensitivity for the considered frequency range can be low.

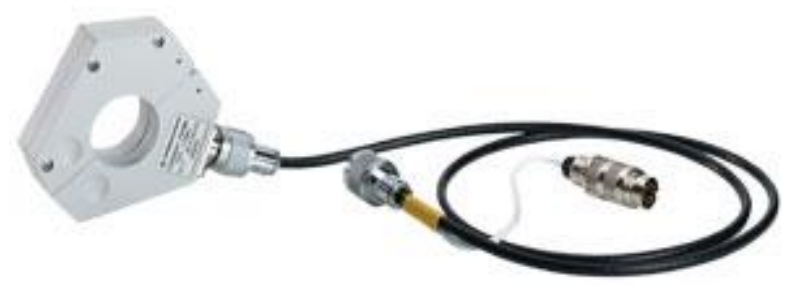

Figure 3: Current Probe R\&S EZ-17

For this reason, current probes based on Hall-sensors can be more interesting. The range starts from DC and commercially available probes give a flat response up to $30 \mathrm{MHz}$ or more. The lowest current capability for commercially available probes is typically some hundreds of $\mu \mathrm{A}$, in combination with a limited dynamic range. An example of a probe based on the Hall- principle is given in Figure 4.

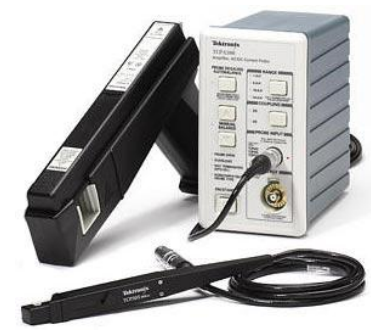

Figure 4: Current probe Tektronix TCPA300

A specific type of field probe is the Rogowski coil. The Rogowski coil is based on Faraday's law of induction:

$V=\oint \vec{E} \overrightarrow{d l}=-\iint \frac{\partial \vec{B}}{\partial t} d \vec{S}$

This law states that the induced voltage $V$ in a closed circuit is proportional to flux change in the total linked flux by the circuit.

Rogowski coils are air-cored coils. The sensor is made of a helical coil, with starts and ends at the same side. This gives the possibility to open the coil to be put around a conductor. Another advantage of this setup is that interference will be cancelled out. Large diameters of coils are available on the market. In this way, large conductors and even common mode currents in shafts can be measured. The air coil results in a low inductance, making a high frequency response possible. Also due to the air coil, there is no saturation and the sensor is highly linear, even for large currents. The main drawback of the sensor is the low sensitivity. The output voltage for commercially available coils is typically $200 \mathrm{mV} / \mathrm{A}$. The coils have a large dynamic range, but a limited sensitivity. For this reason, a dedicated Rogowski coil made for measuring currents below $100 \mu \mathrm{A}$ will be of a homemade type. Rogowski coils can be used from the mHz-range up to some tens of MHz. The coil measures a derivative of the current, meaning that an integrator is necessary (Figure 5).



Figure 5: Basic Rogowski Transducer [12]

\section{Voltage measurements}

Capacitive probes are a voltage measurement method based on a capacitive voltage division. The voltage probe can be seen as a similar method. The capacitive voltage probe (CVP) is allowed by CISPR 22 [5] as an alternative for ISN's (impedance stabilization network). These networks are similar to AMN's, but are specific for data 
cables, e.g. coax or UTP (unshielded twisted pair). The main advantage of the CVP in comparison to other capacitive methods is the built-in amplifier, decreasing and flattening the attenuation to $20 \mathrm{~dB}$. The setup is shown in Figure 6.

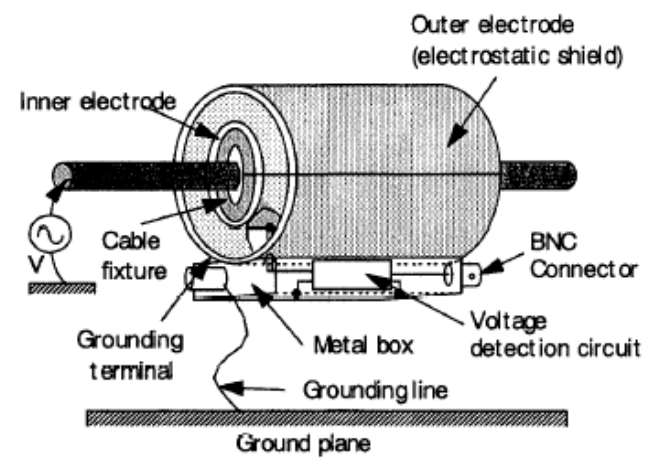

Figure 6: Capacitive Voltage Probe [13]

The CVP is intended to measure between $150 \mathrm{kHz}$ and 30 MHz. Commercially available CVP's show a linear behavior down to $10 \mathrm{kHz}$, which make the probe suitable in the considered range. Nevertheless, this method is not measuring current, but directly a voltage. Research is necessary to see if this type of voltage measurement is sufficiently correlated to the current measurement in the $\mathrm{PCH}$ domain.

\section{E. Time domain vs. frequency domain}

Besides the selection of the probe, a choice of whether time domain measurements or frequency domain measurements are convenient has to be made. Frequency measurements are normally used by all standards considering frequencies above $9 \mathrm{kHz}$. The main benefit is that most spectrum analyzers and EMI receivers are much more sensitive than oscilloscopes with an FFT (by DFT)function (Fast Fourier Transform by Discrete Fourier Transform). On the other hand, spectrum analyzers and similar devices are not designed for measuring low frequencies, which will decrease the sensitivity in the considered range. Most commercially available spectrum analyzers/ EMI receivers have a lower limit of $9 \mathrm{kHz}$. Another drawback of the use of a spectrum analyzer is the lack of phase information, which does can be obtained by an oscilloscope with FFT-function. Besides this, there is limited information on the repetitive character of the measured harmonic. Measurements above $150 \mathrm{kHz}$ use specific types of detectors, e.g. quasi-peak or average detectors. This is not the case for the range beneath $2 \mathrm{kHz}$, where RMS measurements are done. When measuring with an oscilloscope, special attention must go to the adjustment of the time base. This determines the window on which the FFT is performed, and as a consequence, the representation of spectral components depends on it. Finally, oscilloscopes have multiple inputs, where spectrum analyzers and receivers have only one input. When performing comparative measurements, the lack of multiple inputs can be inconvenient.

The choice between time and frequency depends on the area that is investigated. Considering the advantages and the disadvantages of both time domain and frequency domain measurements, in a first approach, the use of a spectrum analyzer is preferred for analyzing $\mathrm{PCH}$, because of the higher accuracy.

\section{Measurements}

\section{A. Current probe}

To compare some of the above mentioned measuring techniques, measurements on a $3 \mathrm{~W}$ CFL were performed. It is known that the input current waveform of CFL's contains harmonics [15], but due to the topology of the lamp, also $\mathrm{PCH}$ are present [2]. In Figure 7 the measurement of the spectrum of the current drawn by the CFL is displayed. A comparison between two different current probes is made: a Tektronix TCPA300 probe and a Rohde \& Schwarz EZ-17 current probe. Both probes deliver a voltage to the measuring receiver, so a conversion of the measured values is needed to obtain the current in Amperes. The Tektronix probe has a fixed conversion factor $(5 \mathrm{~A} / \mathrm{V})$. The conversion with the EZ-17 probe relies on the transfer impedance (2) which depends on the frequency and given by a characteristic in the datasheet of the probe. In Figure 7 the switching frequencies can be clearly seen on approximately $30 \mathrm{kHz}$ and multiples. Also it can be concluded that the noise level of the TCPA300 probe is a few $\mathrm{dB}$ higher than for the EZ-17, but in general the results can be considered as equal. As the measurements are not performed simultaneously (the measuring receiver only has one channel), this can also be an origin of small differences in the results.

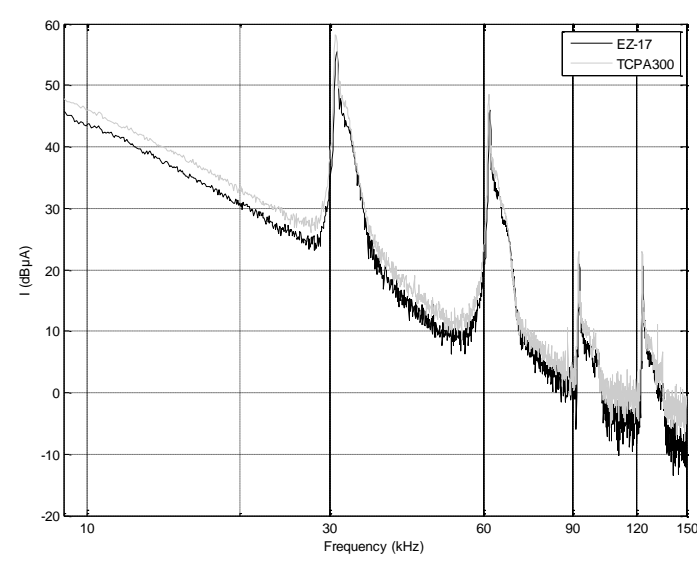

Figure 7: Measurement with two current probes (Blue: TCPA300, red: EZ-17), performed with measuring receiver

\section{B. Rogowski coil}

In Figure 8, two measurements with different Rogowski coils and a measurement with the EZ-17 current probe are shown. From these measurements, it can be seen that the Power Electronic Measurements Ltd. CWT 015 coil has a higher noise level than the CWT 06 coil, conform the datasheets of the probes. The noise level given by the manufacturer of the coils is above the measured current. When increasing the number of Ampere windings, also the noise level increases. As a consequence, Rogowski coils are not the most appropriate measurement 
instrument in this case. The switching harmonics on approx. $90 \mathrm{kHz}$ and $120 \mathrm{kHz}$ even disappear in the noise, while these can be clearly distinguished with the EZ-17 probe. This probe has a much lower noise level than the Rogowski coils.

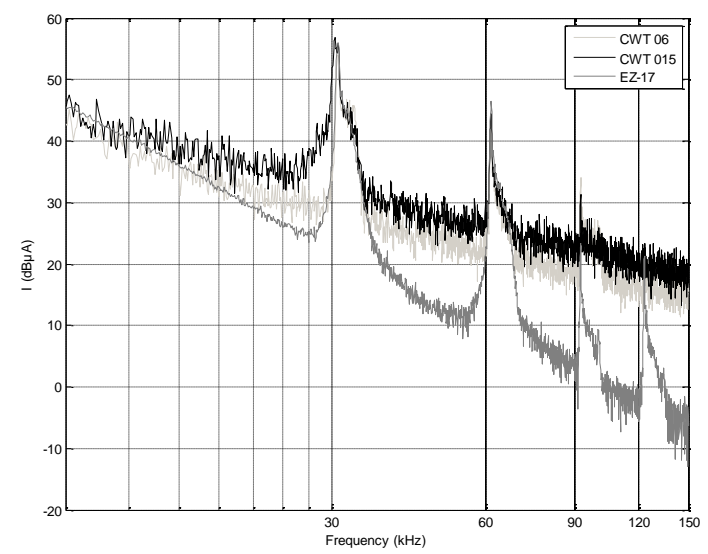

Figure 8: Measurement with two Rogowski coils (black: CWT015, light grey: CWT 06) and EZ-17 current probe (dark grey), performed with EMI receiver

\section{EMI receiver vs. digital scope}

In Figure 9, the current spectrum is measured with the EZ17 current probe, both with a scope (with FFT by DFT) and an EMI receiver. It can be seen that the noise level of the scope is much higher than for the measuring receiver, although the levels of disturbance at the switching harmonics are the same. Due to the high noise level of the scope, the $\mathrm{PCH}$ at approx. $90 \mathrm{kHz}$ and $120 \mathrm{kHz}$ almost disappear in the noise.

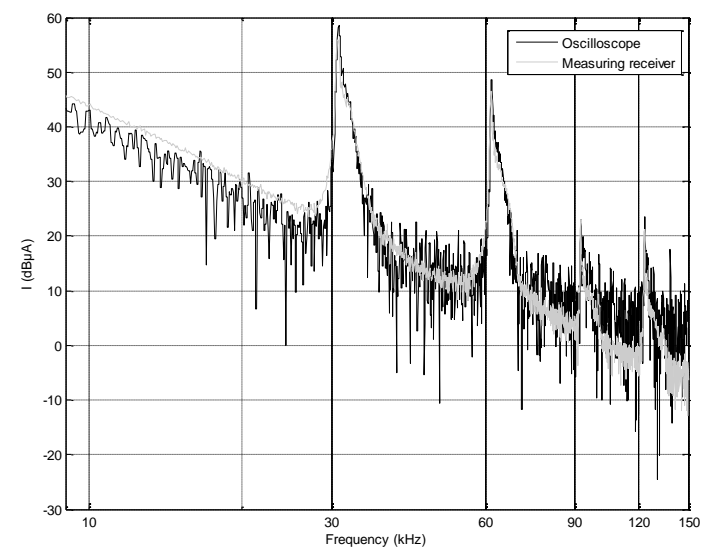

Figure 9: Measurement with the EZ-17 probe (black: scope, grey: EMI receiver)

\section{Conclusion}

Both power quality domain and the EMC domain standards impose standardized measurement methods, but in the zone of $\mathrm{PCH}$, no appropriate measurement methods exist. In this paper, an appropriate measurement method for $\mathrm{PCH}$ is investigated. For the considered frequency range, the use of a spectrum analyzer / EMI receiver is preferred above the use of a scope with FFT (by DFT) function. This because of the higher accuracy and a smaller risk of measuring errors. Also, the use of an AMN is recommended. This provides a defined impedance seen from the EUT. Measurements with two different current probes give the same results, only the noise level differs a little. Measurements with Rogowski coils show a much higher noise level, as expected when considering the datasheets of the coils. As a conclusion, Rogowski coils are not the appropriate measurement method for the small currents drawn by CFL's.

\section{Acknowledgement}

The authors would like to express thanks to EFRO Cleantech for supporting the fund of this research.

\section{References}

[1] J. Kirchof, G. Klein, "EMV - Grenzwertlücke Wechselrichter stört Zähler," presented at 24. Symposium Photovoltaische Solarenergie, Bad Staffelstein, Germany, 2009.

[2] S. K. Rönnberg, "Power line communication and customer equipment," M.S. thesis, Dept. of engineering Sciences and Mathematics, Luleå University of Technology, Luleå, Sweden, 2011.

[3] IEC 61000-3-2 Ed.3, "Electromagnetic compatibility (EMC) - Part 3-2: Limits - Limits for harmonic current emissions (equipment input current $<=16 \mathrm{~A}$ per phase", 2005.

[4] IEC 61000-4-7 Ed. 2, "Electromagnetic compatibility (EMC) - Part 4-7: Testing and measurement techniques General guide on harmonics and interharmonics measurements and instrumentation, for power supply systems and equipment connected thereto", 2002.

[5] CISPR 22 Ed. 6, "Information technology equipment Radio disturbance characteristics - Limits and methods of measurement", 2008.

[6] CISPR 14-1 Ed. 5: "Electromagnetic compatibility Requirements for household appliances, electric tools and similar apparatus - Part 1: Emission”, 2005.

[7] CISPR 15 Ed. 7, "Limits and methods of measurement of radio disturbance characteristics of electrical lighting and similar equipment", 2005.

[8] CISPR 16 -1 Ed. 2.1, "Specification for radio disturbance and immunity measuring apparatus and methods - Part 1: Radio disturbance and immunity measuring apparatus", 2002.

[9] J. Desmet, G. Vanalme, R. Belmans, "Evaluation, practice and remarks on the IEC 61000-3-2 standard", EMC Europe, Sorrento Italy, Sept. 2002

[10] EN50065-1, "Signalling on low-voltage electrical installations in the frequency range $3 \mathrm{kHz}$ to $148,5 \mathrm{kHz}-$ Part 1: General requirements, frequency bands and electromagnetic disturbances", 2011.

[11] IEC 61000-3-8, "Electromagnetic compatibility (EMC) Part 3: Limits - Section 8: Signalling on low-voltage electrical installations - Emission levels, frequency bands and electromagnetic disturbance levels", 1997.

[12] W. F. Ray, C. R. Hewson, "High Performance Rogowski Current Transducers," presented at IEEE Industry Applications Conference, Rome, Italy, October 2000.

[14] J. Knockaert, J. Catrysse, R. Belmans, "Comparison of alternative conducted emission measurement methods by using FSV and IELF algorithms," presented at International symposium on electromagnetic compatibility (EMC Europe 2006), Barcelona, Spain, September 2006 
[15] S. Elphick, P. Ciufo, S. Perera, "Laboratory investigation of the input current characteristics of modern domestic appliances for varying supply voltage conditions," presented at IEEE International Conference on Harmonics and Quality of Power, Bergamo, Italy, 2010. 